\title{
Dynamic state estimation for distribution networks with renewable energy integration
}

\author{
Phuong H. Nguyen ${ }^{\mathrm{a}, \mathrm{b}}$, Ganesh K. Venayagamoorthy ${ }^{\mathrm{b}}$ \\ Wil L. Kling ${ }^{\text {a }}$ Paulo F. Ribeiro ${ }^{\text {a }}$ \\ ${ }^{a}$ Eindhoven University of Technology, Den Dolech 2, Eindhoven, 5600MB, the Netherlands \\ ${ }^{b}$ Real-Time Power and Intelligent Systems Laboratory, Holcombe Department of Electrical and Computer Engineering, \\ Clemson University, 303D Riggs Hall, Clemson, SC 29634-0915, USA
}

\begin{abstract}
The massive integration of variable and unpredictable Renewable Energy Sources (RES) and new types of load consumptions increases the dynamic and uncertain nature of the electricity grid. Emerging interests have focused on improving the monitoring capabilities of network operators so that they can have accurate insight into a network's status at the right moment and predict its future trends. Though state estimation is crucial for this purpose to trigger control functions, it has been used mainly for steady-state analysis. The need for dynamic state estimation (DSE), however, is increasing for real-time control and operation. This paper addresses the important role of DSE over conventional static-state estimation in this new distribution network context. Computational burden mitigates the state-of-the-art utilizations of DSE in real large-scale networks, although DSE was introduced several decades ago. This paper the unscented Kalman filter (UKF) to alleviate computational burden with DSE. The UKF-based approach does not use a linearization procedure and thus outperforms the conventional Extended Kalman Filter based approach to cope with non-linear models. The performance of the UKF method is investigated with a simulation of an 18-bus distribution network on the real-time digital simulator (RTDS) platform. A distribution network with considerable integration of renewable energy production is used to evaluate the UKF-based DSE approach under different types of events.
\end{abstract}

Keywords: Dynamic state estimation, extended Kalman filter, unscented Kalman filter, renewable energy sources, distribution network

\section{Introduction}

Considering the massive integration of variable and unpredictable Renewable Energy Sources (RES) and new types of load consumptions, e.g., heat pumps and electric vehicles, the electricity distribution grid is becoming increasingly complex and dynamic. Real-time control and operation have begun to play an important role in reducing the consequences of intermittency and uncertainty in this new distribution network context. These functions require advanced techniques to not only estimate a system's state variables but also to predict its future trends [1]. By improving the grid's monitoring capabilities, control actions will be triggered in real-time, thus improving system reliability and stability.

Static state estimation (SSE) provides a snapshot of a power system's operating point reflected by state variables, e.g., bus voltage magnitudes and phase angles, based on a set of measurements, such as voltage magnitudes, power flows, and power injections. SSE was first introduced by Schweppe and Wildes in 1970 based on the weighted least square (WLS) [2]. In an effort to reduce computational burden, several hierarchical estimation methods were then proposed, as summarized in [3]. Distributed approaches for SSE also have gained significant interest given their ability to comply with state variables from different

\footnotetext{
* Manuscript received June 15, 2013; revised August 9, 2013.

Corresponding author. Tel.: +31-40-247-4479; E-mail address: p.nguyen.hong @ tue.nl.
} 
network areas on different voltage levels [4]. Different distributed algorithms for SSE were proposed based on the way in which the network areas were defined. In [5], Ebrahimian and Baldick introduced a robust algorithm based on linear augmented Lagrangians for overlapping bus boundaries. Conejo et al. presented a straightforward and effective algorithm for overlapping tie-line boundaries in [6]. Using multi-agent system (MAS) technology, Nordman and Lehtonen proposed a new approach for distributed SSE in [7]. This idea was extended with a completely decentralized SSE method in [8]. More details on static state estimation can be found in [9].

SSE has been utilized widely in the past due to its reliable capability and reasonable accuracy in quasistatic situations. For online and real-time applications, SSE must be repeated in a small enough $\Delta t$ time step (sampling time), which yields undesirable properties [10]. This kind of succession static estimator, also called a tracking estimator algorithm, can provide information only about static steady-state variables. With the highly dynamic nature of today's smart grids, this traditional approach may not be suitable for the advanced real-time control functions desired for complex and uncertain electric power operation.

Dynamic state estimation (DSE) with a relative simplified model for tracking state vectors was also introduced early in 1970 by Debs and Larson in [10]. Leite da Silva et al. extended the approach with a focus on forecasting and filtering the state vectors using exponential smoothing and least-square estimation [11]. Since then, DSE has been known as an alternative state estimation approach that can predict state vectors one time step ahead based on a priori knowledge and that can be corrected with the subsequent measurement sets. Depending on the techniques, estimated DSE variables can be either static state variables, e.g., bus voltage magnitudes and phase angles, or dynamic state variables, e.g., speed variables of generators.

In general, the DSE model is based on the extended Kalman filtering (EKF) theory, which includes the three main steps of parameter identification, state forecasting/prediction, and state filtering/correction. However, EKF must recursively collect time-historic data in order to update the covariance vectors and treat the heavy computation matrices. These steps mitigate the application of EKF in real large-scale power systems.

Recent applications of unscented transformation techniques have significantly improved the performance of Kalman filter-based estimation for DSE. Valverde and Terzija showed the advantage of the unscented Kalman filter (UKF) over the EKF and WLS methods [12]. In [13], the ability of UKF to address dynamic variables, e.g., speed variables and internal generator voltages, was presented with a simplified simulation that included a small number of generators. Other research focusing on estimating the generator variables was introduced in [14]. Recent publications have shown that UKF has advantages in terms of robustness, speed of converge, and identification of bad data compared with the classical EKF-based method.

A UKF-based DSE method for a distribution network with RESs is introduced in this paper. Due to the limitation of measurement devices in the distribution network, the developed method needs to yield reasonable predicted and estimated state variables with much less measurement data than others designed for the transmission system. A detailed model for UKF-based DSE is presented that includes the three main steps of parameter identification, state prediction, and state correction. The paper focuses on the advanced utilization of UKF and adapts this advanced technique across all three steps.

\section{Dynamic State Estimation}

A dynamic model that monitors system operating conditions more completely than static models can be represented by a process equation (1) and a measurement equation (2) as follows:

$$
\begin{aligned}
& \mathbf{x}_{k+1}=\mathbf{f}\left(k, \mathbf{x}_{k}\right)+\mathbf{q}_{k} \\
& \mathbf{y}_{k}=\mathbf{h}\left(k, \mathbf{x}_{k}\right)+\mathbf{r}_{k}
\end{aligned}
$$

where $k$ is the time sample; $\mathbf{x}_{k}$ is the state vector; $\mathbf{q}_{k}$ represents modelling uncertainties corresponding to a white Gaussian noise with zero mean and covariance matrix $\mathbf{Q}_{\mathbf{k}} ; \mathbf{y}_{k}$ is the measurement vector; $\mathbf{h}$ is a set 
of nonlinear load-flow functions for the current network configuration; and $\mathbf{r}_{k}$ is a Gaussian error vector with zero mean and diagonal covariance matrix $\mathbf{R}_{\mathrm{k}}$.

In this dynamic state-space model, equation (1) can be interpreted as the memory of the system state time evolution, and equation (2) is considered its refreshment. Such memory will be responsible for the forecasting capability of the model. Depending on the availability of measurements, the model can be adequate or parsimonious.

The basic idea of a state estimation function is to determine the most likely system state vector $\mathbf{x}$ for either the static steady-state or dynamic state of the system:

$$
\mathbf{x}=\left[\boldsymbol{\theta}_{i}, \mathbf{V}_{i}, \mathbf{P}_{\text {inject }}, \varpi_{i}, \delta_{i}\right]^{T},
$$

based on the quantities measured and acquired by remote terminal units (RTUs), presented as:

$$
\mathbf{y}=\left[\mathbf{V}, \mathbf{P}_{\text {flow }}, \mathbf{Q}_{\text {flow }}, \mathbf{P}_{\text {inject }}, \mathbf{Q}_{\text {inject }}\right]^{T} \text {. }
$$

In general, DSE is achieved by implementing three steps, i.e., parameter identification, state prediction (forecasting), and state correction (filtering), as illustrated in Fig. 1. In the first step, the parameters of the transition function $\mathbf{f}\left(k, \mathbf{x}_{k}\right)$ are identified. This will be associated with the processing noise $\mathbf{q}_{k}$ to predict a step-ahead state variable vector $\tilde{\mathbf{x}}_{k+1}$. Through the measurement function $\mathbf{h}\left(k, \tilde{\mathbf{x}}_{k+1}\right)$ and measurement data $\left(\mathbf{y}_{k+1} ; \mathbf{r}_{k}\right)$, the state vector is corrected as $\hat{\mathbf{x}}_{k+1}$, which will be a fine-grained estimation for use at the control center. While SSE based on WLS provides only a snapshot of the current state vector, i.e., $\mathbf{x}_{k}$ at a certain time $k$, DSE aims not only to provide time-varying solutions but also to predict the system state one step ahead. This is in line with the idea of one-step-ahead prediction recently discussed by Venayagamoorthy et al. in [1], which is crucial for enabling real-time operation and control functions. The following sections explain in detail the DSE process using the conventional EKF method and an advanced UKF method.

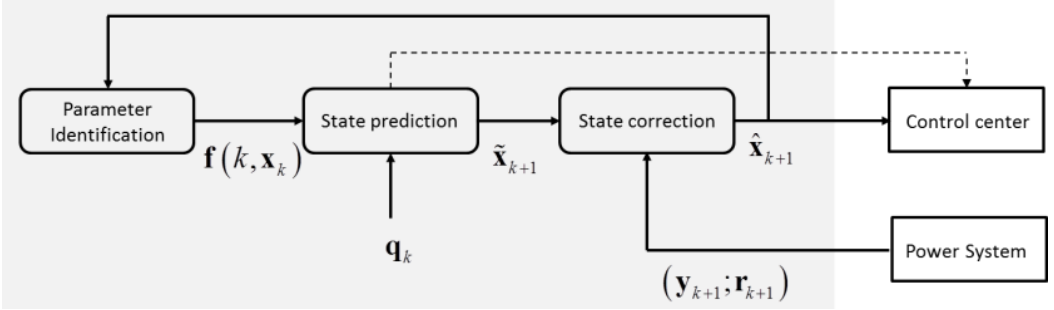

Fig. 1. Dynamic state estimation process

\subsection{EKF-based DSE}

As the most popular approach to handling the complexity of the above model, the EKF-based method simplifies (1) by liberalizing the transit function, assuming the quasi steady-state behavior of the considered system, as follows:

$$
\mathbf{x}_{k+1}=\mathbf{F}_{k} \mathbf{x}_{k}+\mathbf{g}_{k}+\mathbf{q}_{k}
$$

where matrix $\mathbf{F}_{\mathrm{k}}$ represents the speed of transition between states, and vector $\mathbf{g}_{\mathrm{k}}$ is associated with the behavior trends of the state trajectory.

DSE depends heavily on the forecasting technique adopted in [15]. Different forecasting techniques can be applied to estimate $\mathbf{F}_{\mathrm{k}}, \mathbf{g}_{\mathrm{k}}$, and $\mathbf{Q}_{\mathrm{k}}$. The Kalman filter in [16], exponential smoothing in [11], and artificial neural networks (ANN) in [17]-[18] have been utilized successfully under this context.

- Step 1 - Parameter identification

Parameter identification aims to estimate the values of $\mathbf{F}_{\mathrm{k}}, \mathbf{g}_{\mathrm{k}}$, and $\mathbf{Q}_{\mathrm{k}}$ that are used for the state prediction step. Considering an application of Holt's linear exponential smoothing technique, the values 
of $\mathbf{F}_{\mathrm{k}}$ and $\mathbf{g}_{\mathrm{k}}$ can be obtained as follows:

$$
\begin{aligned}
& \mathbf{F}_{k}=\alpha_{k}\left(1+\beta_{k}\right) \mathbf{I} \\
& \mathbf{g}_{k}=\left(1+\beta_{k}\right)\left(1-\alpha_{k}\right) x_{k}^{-}-\beta_{k} \mathbf{a}_{k-1}+\left(1-\beta_{k}\right) \mathbf{b}_{k-1}
\end{aligned}
$$

where $\mathbf{I}$ is the identity matrix, and all associated parameters can be calculated based on a priori knowledge. More details appear in [11]. Despite its rather simple implementation, this technique can offer very short-term predictions (few minutes ahead). However, this linearization step is more suited to quasi steady-state models and may not be suitable for significant dynamic situations.

- Step 2 - State prediction (forecasting)

At this stage, state vector $\tilde{\mathbf{x}}_{k+1}$ is predicted with its covariance matrix $\mathbf{P}_{\tilde{\mathbf{x}}_{k+1}}$ using the following equations:

$$
\begin{aligned}
& \tilde{\mathbf{x}}_{k+1}=\mathbf{F}_{k} \hat{\mathbf{x}}_{k}+\mathbf{g}_{k} \\
& \mathbf{P}_{\tilde{\mathbf{x}}_{k+1}}=\mathbf{F}_{k} \mathbf{P}_{\hat{\mathbf{x}}_{k}} \mathbf{F}_{k}^{T}+\mathbf{Q}_{k} .
\end{aligned}
$$

while $\mathbf{P}_{\hat{\mathbf{x}}_{k}}$ is the covariance matrix to estimate $\hat{\mathbf{x}}_{k}$ at time $k$.

State prediction is an interesting area for the exploitation of computational intelligence (CI). ANN as a typical application of CI has been studied extensively in [17]-[18]. The prediction model can be improved by integrating load forecasting, which was proposed as a forecasting-aided state estimation (FASE) concept in [15].

- Step 3 - State correction (filtering)

By updating a new set of measurements $\mathbf{z}_{k+1}$, the predicted state vector $\tilde{\mathbf{x}}_{k+1}$ can be corrected (filtered), yielding a new state vector $\hat{\mathbf{x}}_{k+1}$ with its error covariance $\mathbf{P}_{\hat{\mathbf{x}}_{k+1}}$. An objective function for the correcting process, at time $k+1$, is presented as follows:

$$
\mathbf{J}(x)=[\mathbf{y}-\mathbf{h}(x)]^{T} \mathbf{R}^{-1}[\mathbf{y}-\mathbf{h}(x)]+[x-\tilde{x}]^{T} \mathbf{P}_{\tilde{x}}^{-1}[x-\tilde{x}]
$$

where the time index $k+1$ has been omitted for simplification, and $\mathbf{R}$ is the variance vector of the measurement errors.

Similar to WLS estimation for SSE, minimizing $\mathbf{J}(x)$ yields an iterative solution, i.e., iterated extended Kalman filter, as follows:

$$
\hat{\mathbf{x}}_{k+1}=\tilde{\mathbf{x}}_{k+1}+\mathbf{K}_{k+1}\left[\mathbf{y}_{k+1}-\mathbf{h}\left(\tilde{x}_{k+1}\right)\right] .
$$

The gain matrix $\mathbf{K}_{k+1}$ is computed using the following equation:

$$
\mathbf{K}_{k+1}=\left[\mathbf{H}^{T} \mathbf{R}^{-1} \mathbf{H}+\mathbf{P}_{\tilde{x}}^{-1}\right]^{-1} \mathbf{H}^{T} \mathbf{R}^{-1}
$$

where $\mathbf{H}_{k+1}=\frac{\partial \mathbf{h}(x)}{\partial x}$ is the Jacobian matrix. With respect to $\hat{\mathbf{x}}_{k+1}$, its error covariance matrix $\mathbf{P}_{\hat{\mathbf{x}}_{k+1}}$ is computed as follows:

$$
\mathbf{P}_{\hat{\mathbf{x}}_{k+1}}=\left[\mathbf{H}^{T} \mathbf{R}^{-1} \mathbf{H}+\mathbf{P}_{\tilde{x}}^{-1}\right]^{-1} .
$$

\subsection{UKF-based DSE}

Basically, EKF is an extension of Kalman filtering that uses a linearization procedure to solve nonlinear models. Though this approach has been considered feasible, it provides only an approximation of the optimal nonlinear estimation. It causes biased estimates and erroneous covariance [12]. 
Furthermore, calculating the Jacobian matrix $\mathbf{H}_{\mathrm{k}+1}$ for each time step could slow down the DSE process.

UKF-based DSE improves the process because it can handle the non-linear nature of DSE. Based on the unscented transformation (UT) theory, the approach propagates a statistical distribution of the state via non-linear equations to provide better results. The three main steps of DSE will be adjusted according to the UT technique as follows:

- Step 1 - Parameter identification

In addition to identifying $\mathbf{F}_{\mathrm{k}}$ and $\mathbf{g}_{\mathrm{k}}$, this stage includes sigma point calculation. From the current state vector $\hat{\mathbf{x}}_{k}$ and its covariance $\mathbf{P}_{\hat{\mathbf{x}}_{k}}$, UT propagates a statistical distribution to form a matrix $\mathbf{X}_{k}$ of $2 N+1$ sigma vectors, as follows:

$$
\mathbf{X}_{k}=\left[\begin{array}{lll}
\hat{\mathbf{x}}_{k} & \hat{\mathbf{x}}_{k} \sqrt{(N+\lambda) \mathbf{P}_{\hat{\mathbf{x}}_{k}}}-\mathbf{x}_{k} \sqrt{(N+\lambda) \mathbf{P}_{\hat{\mathbf{x}}_{k}}}
\end{array}\right]
$$

where $\lambda=\alpha^{2}(N+\kappa)-n$ is a scaling parameter with spreading constant $\alpha\left(10^{-4} \leq \alpha \leq 1\right)$ and secondary scaling $\kappa$ (usually, $\kappa=3-n$ ). Propagating sigma points around the state vector ensures the non-linear nature of the transition function.

- Step 2 - State prediction (forecasting)

From the sets of sigma points in (12), the prediction step in (5) is adjusted as follows:

$$
\begin{aligned}
& \tilde{\mathbf{X}}_{k+1}^{i}=\mathbf{F}_{k} \mathbf{X}_{k}^{i}+\mathbf{g}_{k} \\
& \tilde{\mathbf{x}}_{k+1}=\sum_{i=0}^{2 N} W_{i}^{m} \mathbf{X}_{k}^{i} \\
& \mathbf{P}_{\tilde{\mathbf{x}}_{k+1}}=\sum_{i=0}^{2 N} W_{i}^{c}\left(\tilde{\mathbf{X}}_{k+1}^{i}-\tilde{\mathbf{x}}_{k+i}\right)\left(\tilde{\mathbf{X}}_{k+1}^{i}-\tilde{\mathbf{x}}_{k+i}\right)^{T}+\mathbf{Q}_{k} .
\end{aligned}
$$

with weighting factors given by

$$
W_{0}^{m}=\frac{\lambda}{N+\lambda} ; W_{0}^{c}=\frac{\lambda}{N+\lambda}+1-\alpha^{2}+\beta ; \quad W_{k}^{m}=W_{k}^{c}=\frac{1}{2(N+\lambda)} .
$$

- Step 3 - State correction (filtering)

From predicted state vector $\tilde{\mathbf{x}}_{k+1}$ and its covariance $\mathbf{M}_{k+1}$, a new set of sigma points is generated as:

$$
\hat{\mathbf{X}}_{k+1}=\left[\begin{array}{lll}
\tilde{\mathbf{x}}_{k+1} & \tilde{\mathbf{x}}_{k+1} \sqrt{(N+\lambda) \mathbf{P}_{\tilde{\mathbf{x}}_{k+1}}}-\tilde{\mathbf{x}}_{k+1} \sqrt{(N+\lambda) \mathbf{P}_{\tilde{\mathbf{x}}_{k+1}}}
\end{array}\right]
$$

to be propagated through the following measurement-update equations:

$$
\begin{aligned}
& \hat{\mathbf{Y}}_{k+1}^{i}=\mathbf{h}\left(\hat{\mathbf{X}}_{k+1}^{i}\right) \\
& \hat{\mathbf{y}}_{k+1}=\sum_{i=0}^{2 N} W_{i}^{m} \hat{\mathbf{Y}}_{k+1}^{i} \\
& \mathbf{P}_{\hat{\mathbf{y}}_{k+1}}=\sum_{i=0}^{2 N} W_{i}^{c}\left(\hat{\mathbf{Y}}_{k+1}^{i}-\hat{\mathbf{y}}_{k+i}\right)\left(\hat{\mathbf{Y}}_{k+1}^{i}-\hat{\mathbf{y}}_{k+i}\right)^{T}+\mathbf{R}_{k} \\
& \mathbf{P}_{\tilde{\mathbf{x}}_{k+1} \hat{\mathbf{y}}_{k+1}}=\sum_{i=0}^{2 N} W_{i}^{c}\left(\tilde{\mathbf{X}}_{k+1}^{i}-\tilde{\mathbf{x}}_{k+i}\right)\left(\hat{\mathbf{Y}}_{k+1}^{i}-\hat{\mathbf{y}}_{k+i}\right)^{T}
\end{aligned}
$$

Then, the gain matrix $\mathbf{K}_{k+1}$ is calculated as:

$$
\mathbf{K}_{k+1}=\mathbf{P}_{\tilde{\mathbf{x}}_{k+1} \hat{\mathbf{y}}_{k+1}} \mathbf{P}_{\hat{\mathbf{y}}_{k+1}}^{-1}
$$

The correction of the state vector and its covariance are calculated by the following equations: 


$$
\begin{aligned}
& \hat{\mathbf{x}}_{k+1}=\tilde{\mathbf{x}}_{k+1}+\mathbf{K}_{k+1}\left(\mathbf{y}_{k+1}-\hat{\mathbf{y}}_{k+1}\right) \\
& \mathbf{P}_{\hat{\mathbf{x}}_{k+1}}=\mathbf{P}_{\tilde{\mathbf{x}}_{k+1}}-\mathbf{K}_{k+1} \mathbf{P}_{\mathbf{y}_{k+1}} \mathbf{K}_{k+1}^{T} .
\end{aligned}
$$

\section{Test system and results}

The performance of the UKF was investigated via a real-time simulation on an 18-bus distribution network, as shown in Fig. 2. This test network was modified from the IEEE 34-bus test network [19] with the following simplifications and adjustments [20]:

- Approximately one-third of the distributed loads was placed at the end of the line and two-thirds at one-fourth of the way from the source end;

- Only the three main phase sections were included; the unbalance phase loads were summed up at the root;

- The low-voltage $(0.4 \mathrm{kV})$ network segment was represented by an equivalent load;

- Constant PQ loads were represented by dynamic load models. Constant Z loads were represented by passive resistors and inductors. Constant I loads were neglected;

- Considerable renewable energy production from a $2 \mathrm{MW}$ wind turbine (WT) and 55kW solar photovoltaic (PV) was integrated.

The network model was built on the Real-Time Digital Simulation (RTDS) platform. Network sate before event occurring is summarized in Table 1. To perform the slow dynamics of the system, 50 timesample intervals with a time resolution of $0.08 \mathrm{sec}$. were obtained from the RTDS platform. Voltage magnitude values were measured at bus 1 and bus 18, while active and reactive power flows were measured at one end of each network segment. Active and reactive power injections were measured at buses to which three-phase loads were connected. To achieve realistic measurement data, the values of bus voltages, power flows, and power injections from the simulation included interference from random additive Gaussian noise: $N(0 ; 0.15 \%)$. The steady state of the network before the event was as follows:

Table 1. Network state pre-event

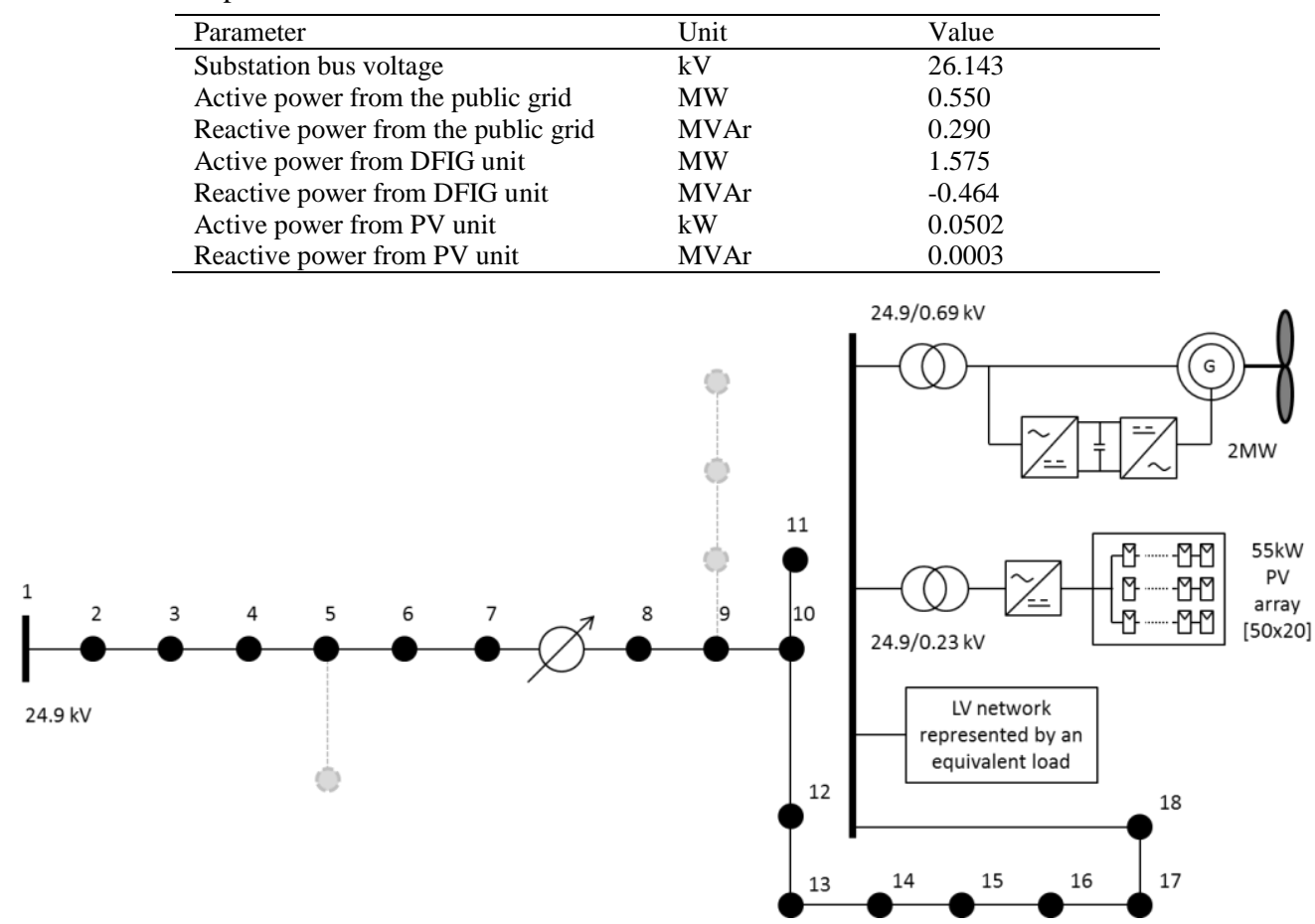

Fig. 2. Single-line diagram of the test network modified from the IEEE 34-bus network. Note that placements of distributed loads will create additional buses in some line sessions. 
To verify the performance of UKF-based DSE, two events were studied in the test network. These events created dynamic changes in the network that the proposed method is expected to capture accurately. Detailed results of these two studied cases appear in the following sections.

\subsection{Case 1 -Low power production from $W T$ and $P V$, together with voltage reduction}

At $t=1 \mathrm{~s}$, the wind speed was reduced from $12.6 \mathrm{~m} / \mathrm{s}$ to $4.4 \mathrm{~m} / \mathrm{s}$, while the irradiation of solar PV decreased from $1029 \mathrm{~W} / \mathrm{m}^{2}$ to $225 \mathrm{~W} / \mathrm{m}^{2}$. This situation was based on one weather sample collected from the real-time power and intelligent systems laboratory's weather station at Clemson, South Carolina, US. The active (in MW) and reactive power (in MVAr) production of the PV and WT units are shown in Fig. 3 along with time (in seconds) simulation. The situation became more equable with a reduction of the bus voltage at the distribution substation (bus 1) from $26.143 \mathrm{kV}$ to $22.41 \mathrm{kV}$, the lowest threshold value ($10 \%)$.
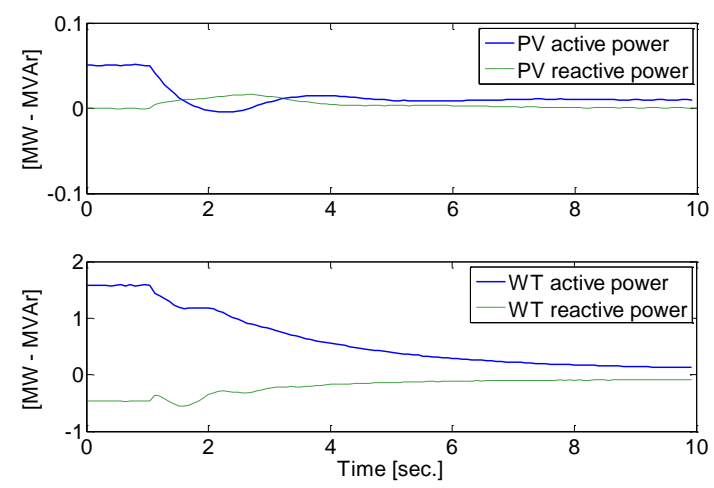

Fig. 3. Reduction of renewable power production due to sudden changes of wind speed and solar irradiation.
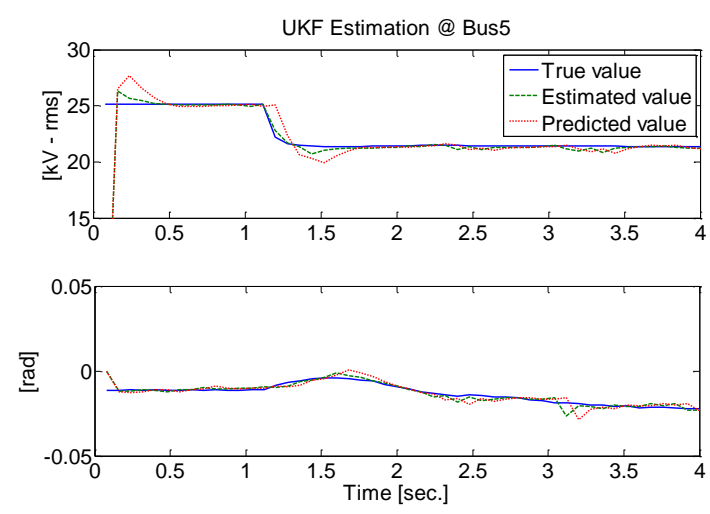

Fig. 4. Comparison of the true bus angle and voltage magnitude value with predicted and estimated values at bus 5 Case 1.

This event was captured by UKF-based DSE with an example of estimated voltage magnitude (in rms $\mathrm{kV}$ ) and bus angle (in radian) together with predicted values at bus 5, as shown in Fig. 4. After some initial time samples, the state vector was predicted quite close to the true value during steady-state operating points. Even with the occurrence of a significant event, this prediction method yielded good results and was able to track new operating points. Fig. 4 also shows how the corrected state vector outperformed the predicted state vector, especially in transient periods of the system.

\subsection{Case 2 - Occurrence of an external fault}

In this case, an external fault occurred at time $t=1.2 \mathrm{~s}$ that caused a serious dip in the voltage at the substation from $26.143 \mathrm{kV}$ to $13.057 \mathrm{kV}$, as shown in Fig. 5. The fault was isolated at $t=2.376 \mathrm{~s}$, thus voltage profile along the feeder was restored. Comparison of the true bus angle (in radian) and voltage 
magnitude (in rms $\mathrm{kV}$ ) value with predicted and estimated values at bus 5 is shown in Fig. 5 . As the figure illustrates, the UKF-based DSE method performed effectively with the dynamic change of the network state.
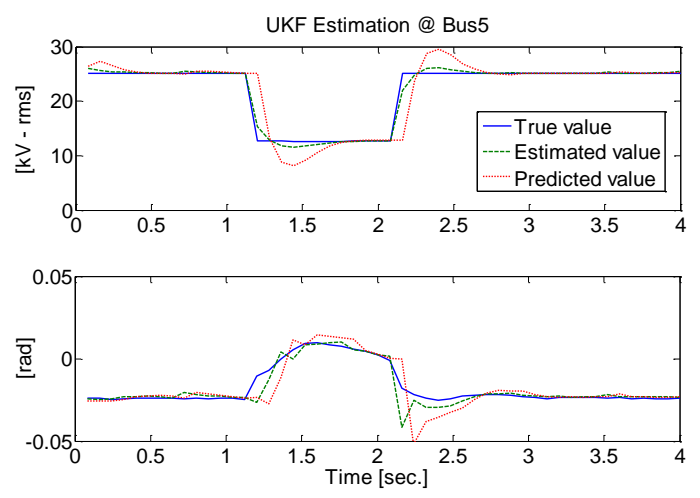

Fig. 5. Comparison of the true bus angle and voltage magnitude value with predicted and estimated values at bus 5 Case 2.

\section{Conclusions}

This paper has addressed the important role of DSE in estimating accurate state variables at the right moment and predicting their trends steps ahead for real-time control and operation. A UKF-based DSE has been introduced to tackle the dynamic nature of the distribution network with increasing RES penetration. The proposed approach requires less computational effort and performs better than the EKFbased method for non-linear models such as DSE. The performance of UKF-based DSE was verified via simulation on an 18-bus distribution test network with considerable wind and solar power integration. Simulation results from two studied events showed the feasibility of the UKF in estimating and predicting the dynamic state of the distribution network. In the simulation, the predicted values were able to track the network's state one step ahead. Depending on the sampling time interval, this prediction can be used to provide advance information for secure operation of the distribution grid.

In future work, a combination of the UKF with Recurrent Neural Networks (RNNs) might be considered to improve DSE. More specifically, the UKF can be used for online training of recurrent neural networks.

\section{Acknowledgements}

The funding provided by the National Science Foundation, USA under the grant ECCS \#1232070 and IIP $\# 1312260$ is gratefully acknowledged

\section{References}

[1] Venayagamoorthy G, Rohrig K, Erlich I. One step ahead: short-term wind power forecasting and intelligent predictive control based on data analytics. IEEE Power and Energy Magazine, 2012; 10(5):70-78.

[2] Schweppe FC, Wildes J. Power system static-state estimation, part I: exact model. IEEE Transactions on Power Apparatus and Systems, 1970; PAS-89(1): 120-125.

[3] Van Cutsem T, Ribbens-Pavella M. Critical survey of hierarchical methods for state estimation of electric power systems. IEEE Transactions on Power Apparatus and Systems, 1983; PAS-102(10):3415-3424.

[4] Shahidehpour M, Yaoyu W. Communication and Control in Electric Power Systems: Applications of Parallel and Distributed Processing. Wiley-IEEE; 2003.

[5] Ebrahimian R, Baldick R. State estimation distributed processing [for power systems]. IEEE Transactions on Power Systems, 2000; 15(4):1240-1246.

[6] Conejo AJ, de La Torre S, Canas M. An optimization approach to multiarea state estimation. IEEE Transactions on Power 
Systems, 2007; 22(1):213-221.

[7] Nordman MM, Lehtonen M. Distributed agent-based state estimation for electrical distribution networks. IEEE Transactions on Power Systems, 2005; 20(2):652-658.

[8] Nguyen PH, Kling WL. Distributed state estimation for multi-agent based active distribution networks. In: Proc. of 2010 IEEE Power and Energy Society General Meeting, 2010:1-7.

[9] Nguyen PH. Multi-agent system based active distribution networks. PhD dissertation. Department of Electrical Engineering, Eindhoven University of Technology. Eindhoven, the Netherlands; 2010.

[10] Debs AS, Larson RE. A dynamic estimator for tracking the state of a power system. IEEE Transactions on Power Apparatus and Systems, 1970; PAS-89(7):1670-1678.

[11] Leite da Silva AM, Do Coutto Filho MB, de Queiroz JF. State forecasting in electric power systems. IEE Proceedings C Generation, Transmission and Distribution, 1983; 130(5):237-244.

[12] Valverde G, Terzija V. Unscented Kalman filter for power system dynamic state estimation. IET Generation, Transmission \& Distribution, 2011; 5(1):29-37.

[13] Wang S, Gao W, Meliopoulos APS. An alternative method for power system dynamic state estimation based on unscented transform. IEEE Transactions on Power Systems, 2012; 27(2):942-950.

[14] Ghahremani F, Kamwa I. Online state estimation of a synchronous generator using unscented Kalman filter from phasor measurements units. IEEE Transactions on Energy Conversion, 2011; 26(4):1099-1108.

[15] Brown M, Coutto D, Cesar J, Souza SD. Forecasting-aided state estimation - part I: panorama. IEEE Transactions on Power Systems, 2009; 24(4):1667-1677.

[16] Schneider KP, Huang Z, Yang B, Hauer M, Nieplocha Y. Dynamic state estimation utilizing high performance computing methods. In: Proc. of IEEE PES Power Systems Conference and Exposition, 2009:1-6.

[17] Sinha AK, Mondal JK. Dynamic state estimator using ANN based bus load prediction. IEEE Transactions on Power Systems, 1999; 14(4):1219-1225.

[18] Balasubramaniam K, Luitel B, Venayagamoorthy GK. A scalable wide area monitoring system using cellular neural networks. In: Proc. of The 2012 International Joint Conference on Neural Networks (IJCNN), 2012:1-8.

[19] Kersting WH. Radial distribution test feeders. Presented at: IEEE Power Engineering Society Winter Meeting, 2001.

[20] Mwakabuta, Sekar A. Comparative study of the IEEE 34 node test feeder under practical simplifications. In Proc. of 39th North American Power Symposium NAPS, 2007:484-491.

[21] Liang J, Venayagamoorthy GK, Harley RG. Wide-area measurement based dynamic stochastic optimal power flow control for smart grids with high variability and uncertainty. IEEE Trans. on Smart Grid, 2012; 3(1):59-69. 